\title{
Pharmacokinetic and Pharmacodynamic Profile of Empagliflozin, a Sodium Glucose Co-Transporter 2 Inhibitor
}

\author{
André J. Scheen
}

Published online: 16 January 2014

(c) The Author(s) 2014. This article is published with open access at Springerlink.com

\begin{abstract}
Empagliflozin is an orally active, potent and selective inhibitor of sodium glucose co-transporter 2 (SGLT2), currently in clinical development to improve glycaemic control in adults with type 2 diabetes mellitus (T2DM). SGLT2 inhibitors, including empagliflozin, are the first pharmacological class of antidiabetes agents to target the kidney in order to remove excess glucose from the body and, thus, offer new options for T2DM management. SGLT2 inhibitors exert their effects independently of insulin. Following single and multiple oral doses (0.5-800 mg), empagliflozin was rapidly absorbed and reached peak plasma concentrations after approximately 1.33-3.0 h, before showing a biphasic decline. The mean terminal half-life ranged from 5.6 to $13.1 \mathrm{~h}$ in single risingdose studies, and from 10.3 to $18.8 \mathrm{~h}$ in multiple-dose studies. Following multiple oral doses, increases in exposure were dose-proportional and trough concentrations remained constant after day 6 , indicating a steady state had been reached. Oral clearance at steady state was similar to corresponding single-dose values, suggesting linear pharmacokinetics with respect to time. No clinically relevant alterations in pharmacokinetics were observed in mild to severe hepatic impairment, or in mild to severe renal impairment and end-stage renal disease. Clinical studies did not reveal any relevant drug-drug interactions with several other drugs commonly prescribed to patients with T2DM, including warfarin. Urinary glucose excretion (UGE) rates were higher with empagliflozin versus placebo
\end{abstract}

\footnotetext{
A. J. Scheen $(\bowtie)$

Division of Diabetes, Nutrition and Metabolic Disorders and Division of Clinical Pharmacology, Department of Medicine, CHU Sart Tilman (B35), University of Liège, B-4000 Liège 1, Belgium

e-mail: andre.scheen@chu.ulg.ac.be
}

and increased with dose, but no relevant impact on 24-h urine volume was observed. Increased UGE resulted in proportional reductions in fasting plasma glucose and mean daily glucose concentrations.

\section{Introduction}

Sodium glucose co-transporter 2 (SGLT2) inhibitors are a new class of drug being developed for the treatment of type 2 diabetes mellitus (T2DM). Sodium glucose co-transporters mediate glucose reabsorption in the kidney $[1,2]$. Approximately $90 \%$ of renal glucose reabsorption occurs in the first segment of the proximal tubule and is mediated by SGLT2, a low-affinity high-capacity transporter, and the remaining $10 \%$ is removed in the distal segment via SGLT1, a high-affinity low-capacity transporter [1, 2]. Inhibition of SGLT2 decreases renal glucose reabsorption, promotes urinary glucose excretion (UGE) and reduces plasma glucose concentrations. Because SGLT2 inhibition occurs through an insulin-independent mechanism, the risk of hypoglycaemia is low [3].

SGLT2 inhibition is also associated with weight loss, caused by a reduction in available calories due to UGE, and a reduction in the mass of both subcutaneous and visceral fat $[4,5]$. Blood pressure-lowering effects are also reported in the labelling documents of SGLT2 inhibitors that have gained regulatory approval $[6,7]$.

Unlike SGLT2, SGLT1 is extensively expressed in the small intestine, where it has a significant role in the absorption of glucose and galactose [1]. High selectivity for SGLT2 versus SGLT1 is important in candidate SGLT2 inhibitors, as inhibition of SGLT1 may result in glucosegalactose malabsorption, causing severe diarrhoea and dehydration [1]. Grempler et al. [8] reported empagliflozin 
had the highest selectivity for SGLT2 over SGLT1 $(>2,500$-fold) compared with other SGLT2 inhibitors (tofogliflozin $>1,875$-fold, dapagliflozin $>1,200$-fold, ipragliflozin $>550$-fold and canagliflozin $>250$-fold) (Table 1). However, recent data suggest that transient inhibition of SGLT1 by candidate SGLT2 inhibitors may reduce intestinal glucose absorption [9-11] and may increase serum glucagon-like peptide-1 and peptide YY $[10,11]$. Nevertheless, the safety implications of SGLT1 inhibition are not yet clear.

Seven SGLT2 inhibitor compounds are known to have reached phase III clinical trials. Of these, marketing applications have been submitted in the USA and European Union (EU) for dapagliflozin, canagliflozin and, most recently, empagliflozin. Dapagliflozin was approved in the EU in 2012, while canagliflozin gained approval from the US FDA in March 2013, and other regulatory approvals are pending. SGLT2 inhibitors are currently targeted as monotherapy for patients with inadequate glycaemic control from diet and exercise, who are unable to use metformin (EU specific), and as an add-on therapy with other glucose-lowering agents, including insulin (EU specific). They may offer additional options as an oral therapy for patients with uncontrolled hyperglycaemia and, potentially, for patients requiring weight reduction.

The subject of this review is empagliflozin (BI 10773; 1-chloro-4-( $\beta$-D-glucopyranos-1-yl)-2-[4-( $(S)$-tetrahydrofuran3-yl-oxy)-benzyl]-benzene; $\mathrm{C}_{23} \mathrm{H}_{27} \mathrm{ClO}_{7}$; molecular weight 450.9; Fig. 1), an orally active, potent and selective inhibitor of SGLT2 being studied for the treatment of patients with T2DM [8, 12], developed by Boehringer Ingelheim and Eli Lilly and Company. Phase III trials of empagliflozin given as monotherapy or in combination with oral antidiabetes drugs or insulin reported statistically significant and clinically relevant improvements in glycaemic control, body weight and systolic blood pressure when compared with placebo and active comparators [13-17]. Adverse events with SGLT2

Table 1 Selectivity of SGLT2 inhibitors for SGLT2 versus SGLT1

\begin{tabular}{llrlll}
\hline Compound & \multicolumn{2}{l}{$\mathrm{IC}_{50}(\mathrm{nM})$} & & $\mathrm{pIC}_{50}(\mathrm{nM})^{\mathrm{a}}$ & \\
\cline { 2 - 3 } & SGLT2 & SGLT1 & & SGLT2 & \multicolumn{1}{l}{ SGLT1 } \\
\hline Empagliflozin & 3.1 & 8,300 & & $8.50 \pm 0.02$ & $5.08 \pm 0.03$ \\
Dapagliflozin & 1.2 & 1,400 & & $8.94 \pm 0.06$ & $5.86 \pm 0.07$ \\
Canagliflozin & 2.7 & 710 & & $8.56 \pm 0.02$ & $6.15 \pm 0.06$ \\
Ipragliflozin & 5.3 & 3,000 & & $8.27 \pm 0.04$ & $5.53 \pm 0.02$ \\
Tofogliflozin & 6.4 & 12,000 & & $8.18 \pm 0.12$ & $4.92 \pm 0.09$ \\
\hline
\end{tabular}

Data taken from Grempler et al. [8] [ $\left.{ }^{14} \mathrm{C}\right]$ alpha-methyl glucopyranoside was used as the substrate

$I C_{50}$ inhibitor concentration at half-maximal response, $p I C_{50}$ $-\log \mathrm{IC}_{50}, S G L T$ sodium glucose co-transporter

${ }^{\text {a }}$ Values expressed as mean \pm standard error of mean inhibitors include increased rates of genital infection and urinary tract infection, which are attributed to elevated urinary glucose levels. More patients on empagliflozin than on placebo reported events consistent with genital infection; however, events consistent with urinary tract infection were comparable in both groups [18]. Empagliflozin is currently progressing through phase III clinical trials, while regulatory decisions are awaited for marketing applications recently submitted in the USA and Europe.

This review examines the pharmacokinetic and pharmacodynamic characteristics of empagliflozin in healthy individuals, and in patients with T2DM treated with empagliflozin monotherapy. An electronic literature search was performed on PubMed to identify relevant studies using the generic name 'empagliflozin' (or the compound identifier 'BI 10773'), without date limits, published as English-language articles. All publications reporting pharmacokinetic and/or pharmacodynamic data on empagliflozin/BI 10773 in humans were considered for this review. Furthermore, the conference proceedings websites of the American Diabetes Association and the European Association for the Study of Diabetes were searched for abstracts of relevant non-published empagliflozin trials. Additional information on empagliflozin was provided by the manufacturers.

\section{Clinical Pharmacokinetics}

Pharmacokinetic characteristics of empagliflozin in healthy subjects and in patients with T2DM are summarised in Table 2 (see also Figs. 2, 3). The pharmacokinetic data from patients with T2DM were similar to those obtained in healthy volunteers.

\subsection{Healthy Subjects}

In a single rising oral doses $(0.5-800 \mathrm{mg})$ study in healthy subjects, empagliflozin was rapidly absorbed after oral administration and showed a biphasic decline [19]. Increases in exposure, as measured by the area under concentrationtime curve (AUC) of analyte in plasma over time interval from $0 \mathrm{~h}$ extrapolated to infinity $\left(\mathrm{AUC}_{\infty}\right)$ and the maximum plasma concentration $\left(C_{\max }\right)$ were approximately

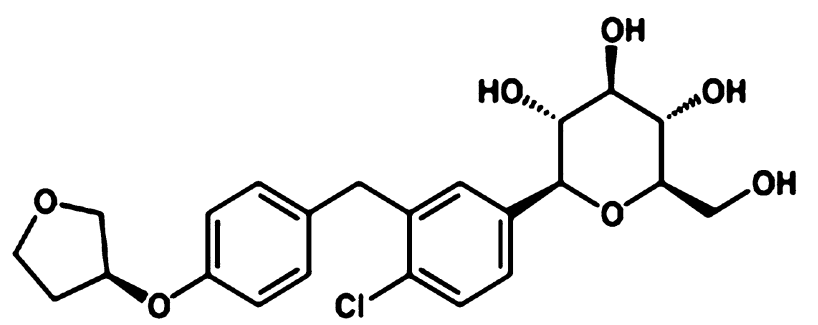

Fig. 1 Structural formula of empagliflozin 


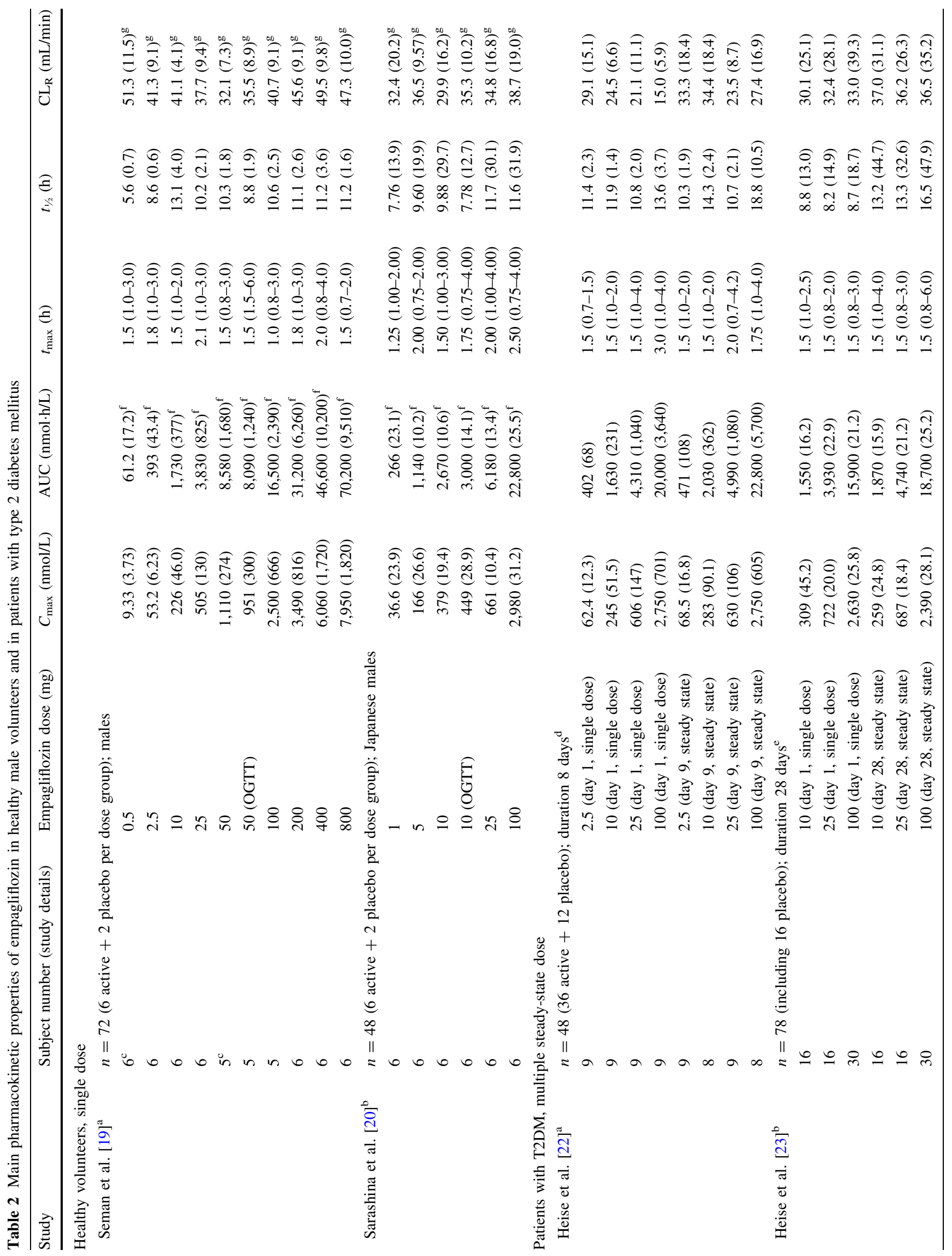




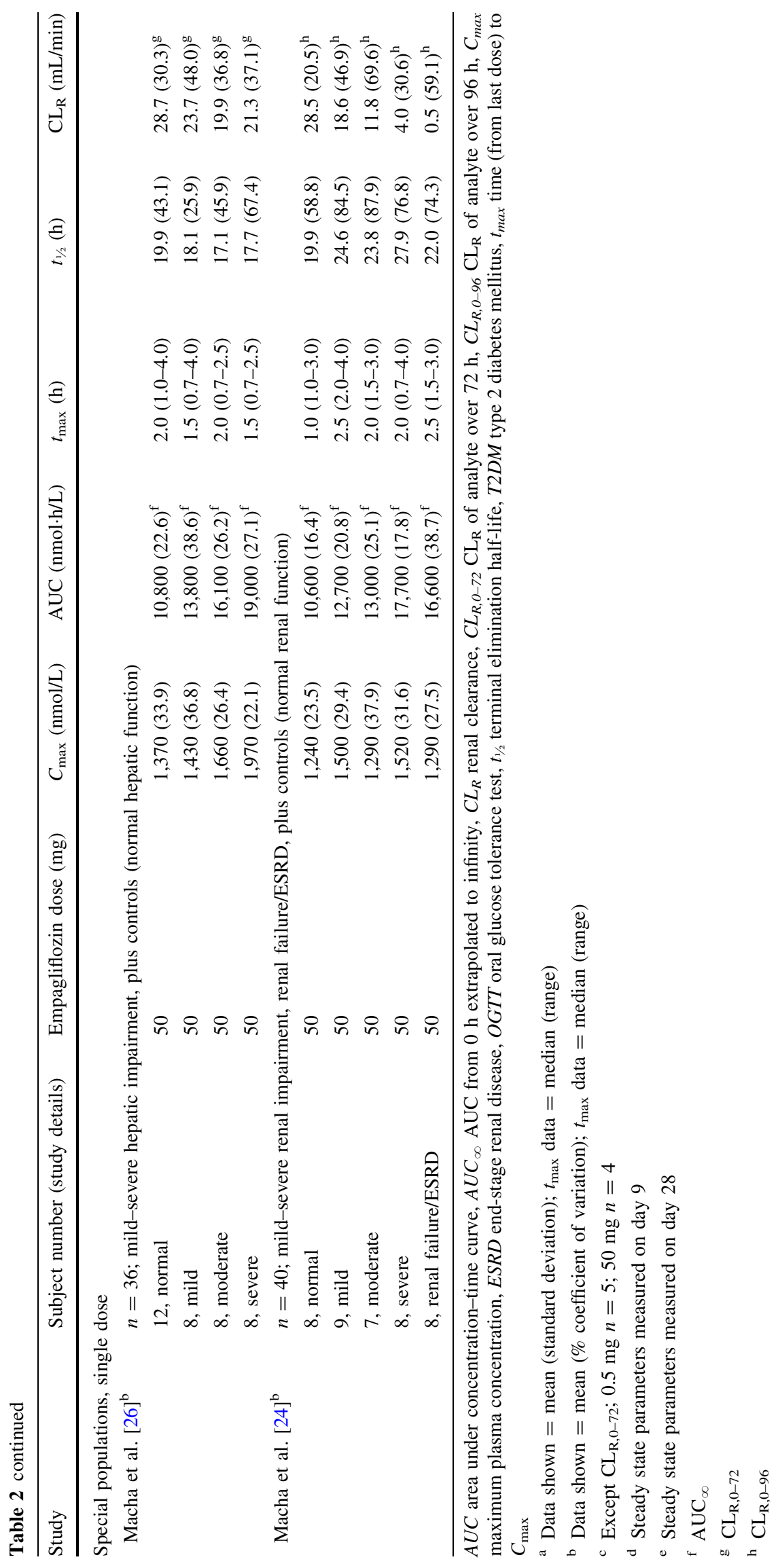


Fig. 2 Clinical

pharmacokinetic and

pharmacodynamic properties of empagliflozin in healthy

volunteers. Results are

expressed as means $( \pm$ standard

deviation for $\mathrm{AUC}_{\infty}$ ) (adapted

from Seman et al. [19]).

Increases in exposure $\left(\mathrm{AUC}_{\infty}\right)$

(upper panel) in healthy

subjects were dose-proportional

over the range of empagliflozin

doses used in this single rising-

dose study. With the exception

of the $200 \mathrm{mg}$ dose, the amount

of $\mathrm{UGE}_{0-24 \mathrm{~h}}$ (lower panel)

increased with increasing dose

of empagliflozin, and maximum

UGE $(90.8 \mathrm{~g})$ occurred at the

$400 \mathrm{mg}$ dose. $A U C_{\infty}$ area under

concentration-time curve of

analyte in plasma over time

interval from $0 \mathrm{~h}$ extrapolated to

infinity, $U G E_{0-24 h}$ urinary

glucose excretion over $24 \mathrm{~h}$
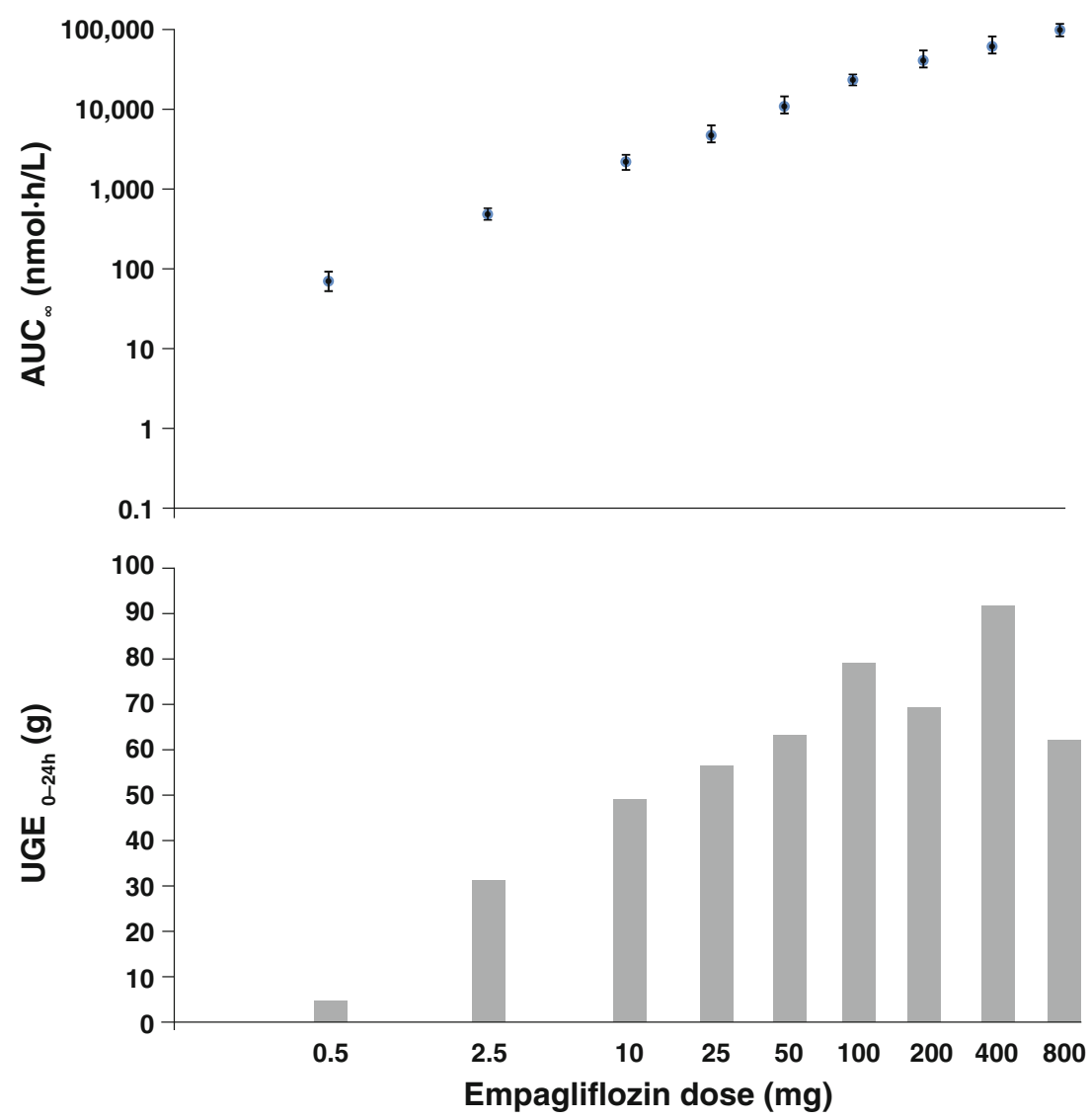

dose-proportional over the range of empagliflozin doses of 0.5-800 mg (Fig. 2, upper panel). Median time to reach $C_{\max }\left(t_{\max }\right)$ ranged from 1.5 to $2.1 \mathrm{~h}$ and the terminal elimination half-life $\left(t_{1 / 2}\right)$ was up to $13.1 \mathrm{~h}$ [19]. Similar results were reported in a single rising oral doses (1-100 mg) study of healthy Japanese males [20]. Exposure was found to be approximately 1.5 -fold higher in Japanese subjects than Caucasians, probably due to the lower body weight in the Japanese group (62.6 vs. $79.0 \mathrm{~kg}$ ) [20].

Mean plasma concentration-time profiles of empagliflozin were similar under fasted and fed conditions (Table 2) [19]. Administration with food resulted in a slightly delayed absorption of empagliflozin, and both mean $\mathrm{AUC}_{\infty}$ and $C_{\max }$ were lower under fed conditions than when the drug was given in the fasted condition: the geometric mean ratios (GMR; \%) and $90 \%$ confidence intervals (CI) for $\mathrm{AUC}_{\infty}$ and $C_{\max }$ were 89.8 (84.5-95.5) and 70.7 (61.0-81.8), respectively [19]. The authors commented that this appeared to be due to an effect on the rate of absorption of empagliflozin, rather than on the extent of its absorption, which was within the usual bioequivalence criteria, and that the findings were consistent with the high aqueous solubility of empagliflozin (reported as unpublished data) [19]. This was not considered clinically significant, and it was concluded that empagliflozin can be administered independently of food. This was supported by the results of a food effect study [21].

Renal clearance $\left(\mathrm{CL}_{\mathrm{R}}\right)$ over $72 \mathrm{~h}$ ranged from 32.1 to $51.3 \mathrm{~mL} / \mathrm{min}$, and the cumulative fraction of empagliflozin excreted in urine $\left(f_{\mathrm{e}}\right)$ over $72 \mathrm{~h}$ ranged from 11.0 to $18.7 \%$ [19]. Plasma concentrations of empagliflozin at higher doses (>100 mg) were generally detectable for $72 \mathrm{~h}$, while plasma concentrations of empagliflozin fell below the limit of quantification [lower limit for plasma $1.11 \mathrm{nmol} / \mathrm{L}$ $(0.5 \mathrm{ng} / \mathrm{mL})]$ after $24 \mathrm{~h}$ at the $0.5 \mathrm{mg}$ dose [19]. In the Japanese study, empagliflozin was detected in the urine of all dose groups and remained measureable up to $72 \mathrm{~h}$ (with one exception in the $1 \mathrm{mg}$ group) [20]. The $\mathrm{CL}_{\mathrm{R}}$ and $f_{\mathrm{e}}$ over $72 \mathrm{~h}$ were generally similar between dose groups (29.9-38.7 $\mathrm{mL} / \mathrm{min}$, and 21.3-22.9\%, respectively) [20].

\subsection{Patients with Type 2 Diabetes Mellitus}

Following multiple oral doses in patients with T2DM (2.5-100 mg once daily over 8 days [22], or 10-100 mg once daily over 28 days [23]), empagliflozin was rapidly absorbed after oral administration, reaching $C_{\max }$ after 1.33-3.0 h, and then declining in a biphasic fashion, with a mean $t_{1 / 2}$ ranging from 10.3 to $18.8 \mathrm{~h}[22,23]$. Increases in exposure were dose-proportional following multiple oral doses [22, 23], and after multiple doses, trough 
Fig. 3 Clinical

pharmacokinetic and pharmacodynamic properties of empagliflozin in patients with T2DM. Results are expressed as means (adapted from Heise et al. [23]). Increases in empagliflozin exposure $\left(\mathrm{AUC}_{24 h}\right)$ in patients with T2DM were dose-proportional following multiple oral doses. UGE increased in empagliflozin dose groups at day 1 and this was maintained after multiple doses; however, almost no change was observed in the placebo group. MDG decreased on day 1 versus placebo, and dropped further by day 27 . $A U C_{24 h}$ area under concentration-time curve of analyte in plasma over $24 \mathrm{~h}$, $M D G$ mean daily glucose, $T 2 D M$ type 2 diabetes mellitus, $U G E_{O-24 h}$ urinary glucose excretion over $24 \mathrm{~h}$
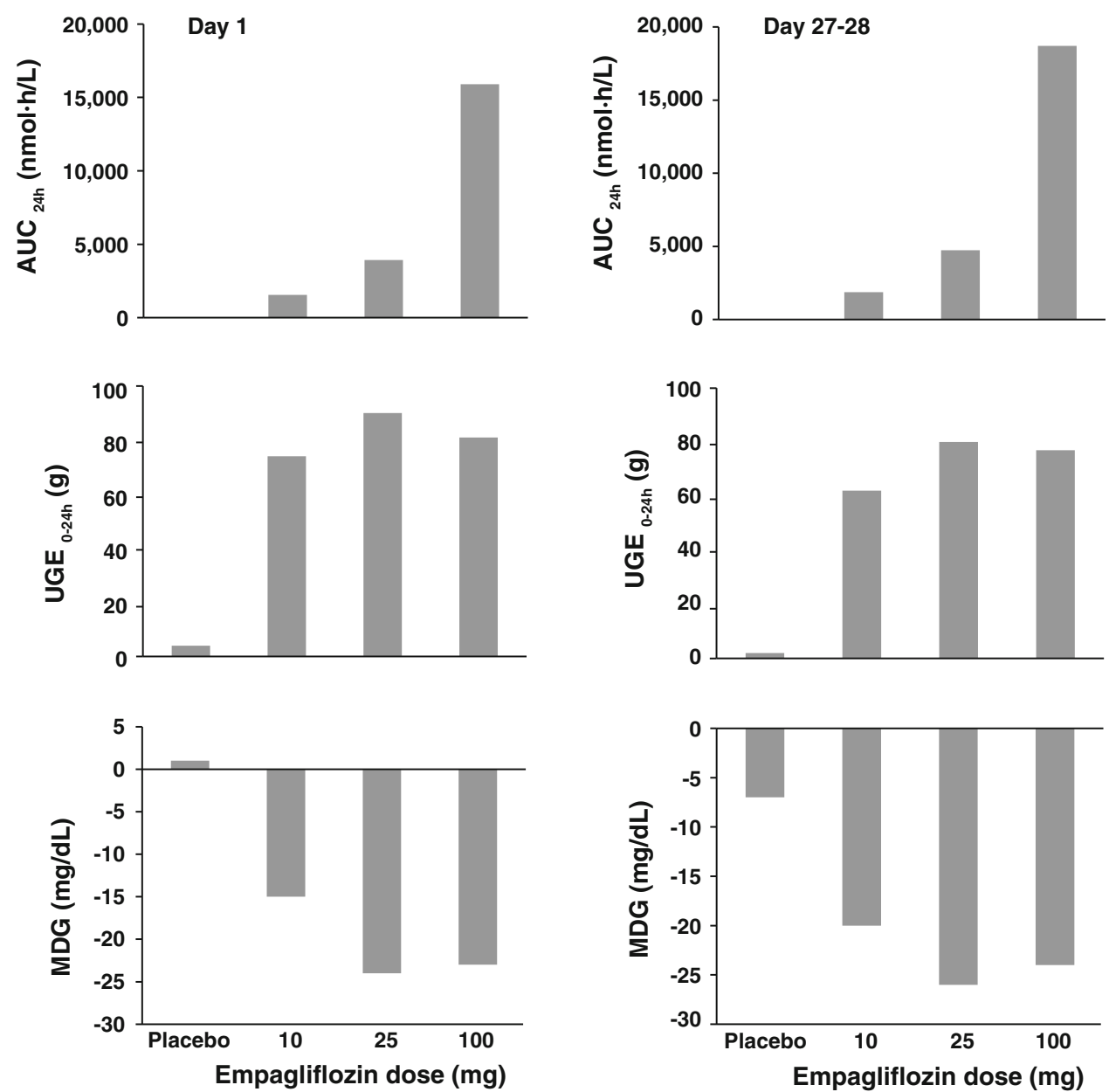

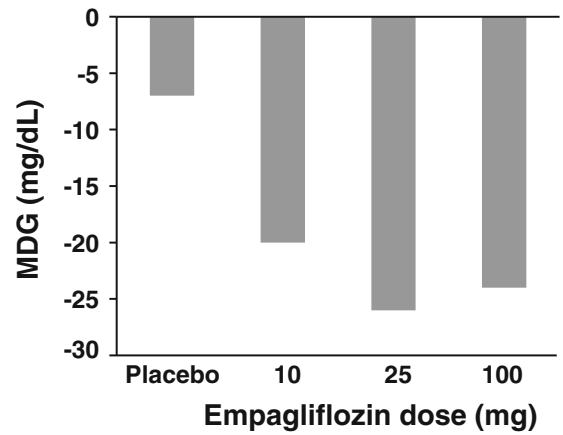

concentrations remained constant after day 6, indicating a steady state had been reached (Fig. 3) [23]. Oral clearance $(\mathrm{CL} / F)$ at steady state was similar to corresponding singledose values, suggesting linear pharmacokinetics with respect to time [22, 23], and approximately $18 \%$ of administered drug was excreted unchanged in the urine [23].

\section{Clinical Pharmacodynamics}

\subsection{Effects on Urinary Glucose Excretion}

The total amount of glucose excreted in the urine was higher with all doses of empagliflozin than with placebo in the single rising-dose study [19]. The mean amount of glucose excreted in urine over the first $24 \mathrm{~h}$ after oral administration of empagliflozin was $3.1 \mathrm{~g}$ with the $0.5 \mathrm{mg}$ dose and $61.6 \mathrm{~g}$ with the $800 \mathrm{mg}$ dose (vs. $0.06 \mathrm{~g}$ with placebo); the maximum UGE was $90.8 \mathrm{~g}$, occurring at the $400 \mathrm{mg}$ dose (Fig. 2, lower panel) [19]. Empagliflozin inhibited reabsorption of $<40 \%$ of filtered glucose at single daily doses of $0.5-10 \mathrm{mg}$, rising to $40-60 \%$ inhibition of filtered glucose at higher doses, and reaching a plateau at around the
$100 \mathrm{mg}$ dose [19]. The total amount of glucose excreted over $72 \mathrm{~h}$ was dose proportional, again reaching a plateau at around the $100 \mathrm{mg}$ dose. The time to maximum rate of UGE was similar in all dose groups (approximately $7 \mathrm{~h}$ ). Food had no relevant effects on UGE when administered with empagliflozin: mean [standard deviation (SD)] cumulative UGE over $24 \mathrm{~h}$ following oral administration of empagliflozin $50 \mathrm{mg}$ was $71.7 \mathrm{~g}$ (13.6) under fasted conditions versus $75.9 \mathrm{~g}$ (17.9) under fed conditions [19].

The single rising doses study in healthy Japanese males reported similar results: higher UGE occurred for all doses of empagliflozin versus placebo, and increased with rising empagliflozin dose [20]. UGE in the $24 \mathrm{~h}$ post-empagliflozin dosing period was similar between Japanese and Caucasian subjects [19] at doses tested in both studies (10 mg, $25 \mathrm{mg}, 100 \mathrm{mg}$ ); thus, the difference in empagliflozin exposure between Japanese and Caucasians (as mentioned in Sect. 2.1) may not affect the expected therapeutic dose [20]. In addition, no significant differences in mean cumulative urine volume were observed in empagliflozin versus placebo in the first $24 \mathrm{~h}(3.11-3.98 \mathrm{~L}$ and $3.17 \mathrm{~L}$, respectively), or in the first $72 \mathrm{~h}(7.12-8.71 \mathrm{~L}$ and $7.30 \mathrm{~L}$, respectively) [20]. 
After administration of a single dose of empagliflozin to patients with T2DM, cumulative UGE over $24 \mathrm{~h}$ ranged from 46.3 to $89.8 \mathrm{~g}$ with empagliflozin versus $5.84 \mathrm{~g}$ with placebo, and similar results were observed after multiple doses [22]. In a separate study of T2DM patients, the mean change in UGE from baseline to the end of the treatment period (day 27) ranged from 64.37 to $78.37 \mathrm{~g}$ for empagliflozin versus $-0.67 \mathrm{~g}$ for placebo (Fig. 3) [23]. Increased UGE and inhibition of renal tubular glucose reabsorption rate were maintained after multiple doses at day 27 compared with baseline, and oral glucose tolerance test (OGTT) on day 28 did not affect the size of the change in UGE from baseline [23]. No clinically relevant changes in urine volume were observed [22, 23].

\subsection{Effects on Plasma Glucose Concentration}

Plasma glucose concentrations were similar in healthy subjects treated with any dose of empagliflozin or with placebo, which is to be expected [19, 20].

The 8-day multiple rising-dose study in patients with T2DM revealed that mean fasting plasma glucose (FPG) decreased by $17.2-25.8 \%$ with empagliflozin versus $12.7 \%$ with placebo [22]. The decrease in mean daily glucose (MDG) was significantly different compared with placebo with the 2.5 and $10 \mathrm{mg}$ doses (MDG: -13.5 $\mathrm{mg} / \mathrm{dL}$ for placebo vs. -29.0 and $-37.0 \mathrm{mg} / \mathrm{dL}$ for empagliflozin 2.5 and $10 \mathrm{mg}$, respectively; $P<0.05$ and $P<0.01$, respectively) [22]. The 28-day multiple-dose study in patients with T2DM reported significant reductions from baseline in mean FPG and MDG in empagliflozin versus placebo (see Fig. 3) [23]. Similar reductions in post-OGTT plasma glucose were seen on OGTT days: differences from placebo on day 28 were statistically significant $(P<0.05$ based on least square means for not-baseline-corrected values) [23]. On day 28, a decrease in glycosylated haemoglobin $\left(\mathrm{HbA}_{1 \mathrm{c}}\right)$ from baseline was observed in all empagliflozin groups; however, the reductions were not statistically significant versus placebo $\left(\mathrm{HbA}_{1 \mathrm{c}}:-0.18 \%\right.$ for placebo; -0.27 , $-0.22,-0.36 \%$ for empagliflozin $10,25,100 \mathrm{mg}$, respectively) [23]. However, the study duration was too short to fully evaluate this parameter.

\section{Pharmacokinetics and Pharmacodynamics in Special Populations}

\subsection{Renal Impairment}

The effect of renal impairment on the pharmacokinetics of empagliflozin was investigated in an open-label, parallelgroup study of 40 subjects with varying degrees of renal impairment, who received a single dose of empagliflozin
$50 \mathrm{mg}$ [24]. Subjects were grouped according to estimated glomerular filtration rate (eGFR): eight subjects had T2DM and normal renal function (eGFR $>90 \mathrm{~mL} / \mathrm{min} / 1.73 \mathrm{~m}^{2}$; control group); nine had T2DM and mild renal impairment (eGFR 60-89 mL/min/1.73 $\mathrm{m}^{2}$ ); seven had T2DM and moderate renal impairment (eGFR $30-59 \mathrm{~mL} / \mathrm{min} /$ $1.73 \mathrm{~m}^{2}$ ); eight had severe renal impairment (eGFR $<30 \mathrm{~mL} / \mathrm{min} / 1.73 \mathrm{~m}^{2}$ ), four of whom also had T2DM; and eight subjects had renal failure/end-stage renal disease (ESRD) requiring dialysis, none of whom had T2DM [24]. Results demonstrated that empagliflozin was rapidly absorbed after oral administration in all subjects, although rate of absorption was slightly slower in subjects with renal impairment than in those with normal renal function, with a median $t_{\max }$ of 2.0-2.5 and $1.0 \mathrm{~h}$, respectively [24]. After reaching $C_{\max }$, plasma drug concentrations declined in a biphasic fashion, which is again consistent with previous reports in healthy subjects and patients with T2DM [24]. Empagliflozin plasma concentrations were similar in subjects with normal renal function and renal impairment. Empagliflozin $\mathrm{AUC}_{\infty}$ values increased by approximately 18, 20, 66 and $48 \%$ in subjects with mild, moderate and severe renal impairment, and renal failure/ESRD, respectively, in comparison with healthy subjects (Table 2), which was attributed to decreased $\mathrm{CL}_{\mathrm{R}}$ [24]. There were also decreases in the mean fraction of the dose excreted in urine following drug administration from time zero to $96 \mathrm{~h}$ $\left(f e_{0-96}\right)$ with increasing renal impairment [24]. The pharmacokinetic results of this study indicated that no dose adjustment of empagliflozin was required in patients with renal impairment [24], which may represent an additional clinical value compared to other glucose-lowering agents currently used in patients with T2DM [25].

The cumulative amount of UGE decreased with increasing renal impairment [24]. Total UGE (change from baseline) over $24 \mathrm{~h}$ was $97.6 \mathrm{~g}$ in subjects with normal renal function, $61.6 \mathrm{~g}, 55.7 \mathrm{~g}$ and $18.2 \mathrm{~g}$ in those with mild, moderate and severe renal impairment, respectively, decreasing to approximately $0.8 \mathrm{~g}$ in subjects with severe renal failure/ESRD [24]. The decrease in UGE followed the same pattern as the decreases in the empagliflozin $\mathrm{CL}_{\mathrm{R}}$ with increasing renal impairment [24].

\subsection{Hepatic Impairment}

The effect of hepatic impairment on the pharmacokinetics of empagliflozin was investigated in an open-label, parallel-group study of 36 subjects (eight subjects each with mild, moderate or severe hepatic impairment per ChildPugh classification, plus 12 matched controls with normal hepatic function) who received a single dose of empagliflozin $50 \mathrm{mg}$ [26]. Results demonstrated that empagliflozin was rapidly absorbed and, after reaching $C_{\max }$, plasma drug 
concentrations declined in a biphasic fashion, which is consistent with previous reports in healthy subjects and patients with T2DM. Compared with subjects with normal hepatic function, GMR of $\mathrm{AUC}_{\infty}$ and $C_{\max }$ were only modestly increased (Table 2). As the increase in empagliflozin exposure was less than twofold in patients with impaired liver function, no dose adjustment of empagliflozin was required in these patients [26].

No relevant differences were reported in mean UGE from drug administration between subjects with hepatic impairment and subjects with normal hepatic function [26]. Mean UGE over $24 \mathrm{~h}$ was $42.6 \mathrm{~g}$ in subjects with normal hepatic function, and $36.2 \mathrm{~g}, 38.4 \mathrm{~g}$ and $40.2 \mathrm{~g}$ in subjects with mild, moderate and severe hepatic impairment, respectively [26].

\section{Drug-Drug Interaction Studies}

A summary of drug-drug interaction studies with empagliflozin is presented in Table 3.

Empagliflozin was coadministered with other medications commonly used in patients with T2DM; specifically, oral glucose-lowering agents (metformin, sitagliptin, linagliptin and glimepiride), an anticoagulant (warfarin), cardiovascular/antihypertensive compounds (diuretics, verapamil, ramipril and digoxin), a cholesterol-lowering agent (simvastatin) and an oral contraceptive (ethinylestradiol/levonorgestrel). These drug-drug interaction studies were evaluated in open-label, randomised, crossover studies in healthy volunteers, with the exception of the study using diuretics, which was performed in patients with T2DM [27].

\subsection{Coadministration with Glucose-Lowering Agents}

\subsubsection{Metformin}

Metformin was found to have no clinically relevant effect on the pharmacokinetics of empagliflozin $50 \mathrm{mg}$ [28]. Similarly, empagliflozin had no clinically relevant effect on the pharmacokinetics of metformin [28]. The $\mathrm{CL}_{\mathrm{R}}$ of empagliflozin and metformin were unaffected by coadministration: $\mathrm{CL}_{\mathrm{R}}$ at steady state was 34.5 versus 36.3 $\mathrm{mL} / \mathrm{min}$ for empagliflozin alone and for coadministration with metformin, respectively, and was 551 versus $523 \mathrm{~mL} / \mathrm{min}$ for metformin alone and for coadministration with empagliflozin, respectively [28]. Furthermore, increased UGE was observed after administration of empagliflozin alone and in combination with metformin: mean (SD) cumulative amounts of glucose recovered after oral administration of empagliflozin $50 \mathrm{mg}$ once daily with and without metformin 1,000 mg twice daily were 62.0 (12.8) and 67.8 (12.6) g, respectively [28]. The results supported the coadministration of empagliflozin and metformin without dose adjustment.

\subsubsection{Sitagliptin}

Coadministration of sitagliptin $100 \mathrm{mg}$ with empagliflozin $50 \mathrm{mg}$ had no clinically relevant effect on the pharmacokinetics of empagliflozin or sitagliptin [29]. Increased UGE was observed after administration of empagliflozin alone and in combination with sitagliptin [29]. Empagliflozin and sitagliptin could be coadministered without dose adjustments.

\subsubsection{Linagliptin}

Linagliptin total exposure and peak exposure were unaffected by coadministration of empagliflozin $50 \mathrm{mg}$ [30]. Empagliflozin total exposure was unaffected by coadministration of linagliptin $5 \mathrm{mg}$; however, there was a slight reduction in empagliflozin peak exposure at steady state [ $C_{\text {max,ss }}$ GMR 0.88 (90 \% CI 0.79-0.99)] when linagliptin was coadministered that was not considered clinically meaningful [30]. Increased UGE was observed after administration of empagliflozin alone and in combination with linagliptin: mean (SD) cumulative UGE over $24 \mathrm{~h}$ after oral administration of multiple doses of empagliflozin $50 \mathrm{mg}$ once daily with and without linagliptin $5 \mathrm{mg}$ were 54.8 (11.2) and 67.2 (14.6) g, respectively [30]. Trough dipeptidyl peptidase-4 inhibition in plasma at steady state $24 \mathrm{~h}$ after drug administration $\left(E_{24, \mathrm{ss}}\right)$ was similar when linagliptin was administered alone or with empagliflozin (median $E_{24, \mathrm{ss}} 83.7$ vs. $83.9 \%$ ) [30]. It was concluded that empagliflozin and linagliptin could be coadministered without dose adjustments.

\subsubsection{Glimepiride}

Coadministration of glimepiride $1 \mathrm{mg}$ with empagliflozin $50 \mathrm{mg}$ was found to have no clinically relevant effects on the pharmacokinetics of empagliflozin or glimepiride [31]. Increased UGE was observed after administration of empagliflozin alone and in combination with glimepiride; mean (SD) cumulative UGE over $24 \mathrm{~h}$ after oral administration of empagliflozin $50 \mathrm{mg}$ once daily with and without glimepiride $1 \mathrm{mg}$ was 72.7 (14.4) and 68.7 (12.3) g, respectively [31]. Based on these data, empagliflozin and glimepiride could be coadministered without dose adjustments.

\subsection{Coadministration with Anticoagulant (Warfarin)}

Warfarin had no effect on empagliflozin exposure or $C_{\max }$ values [32]. Similarly, empagliflozin $25 \mathrm{mg}$ once daily 


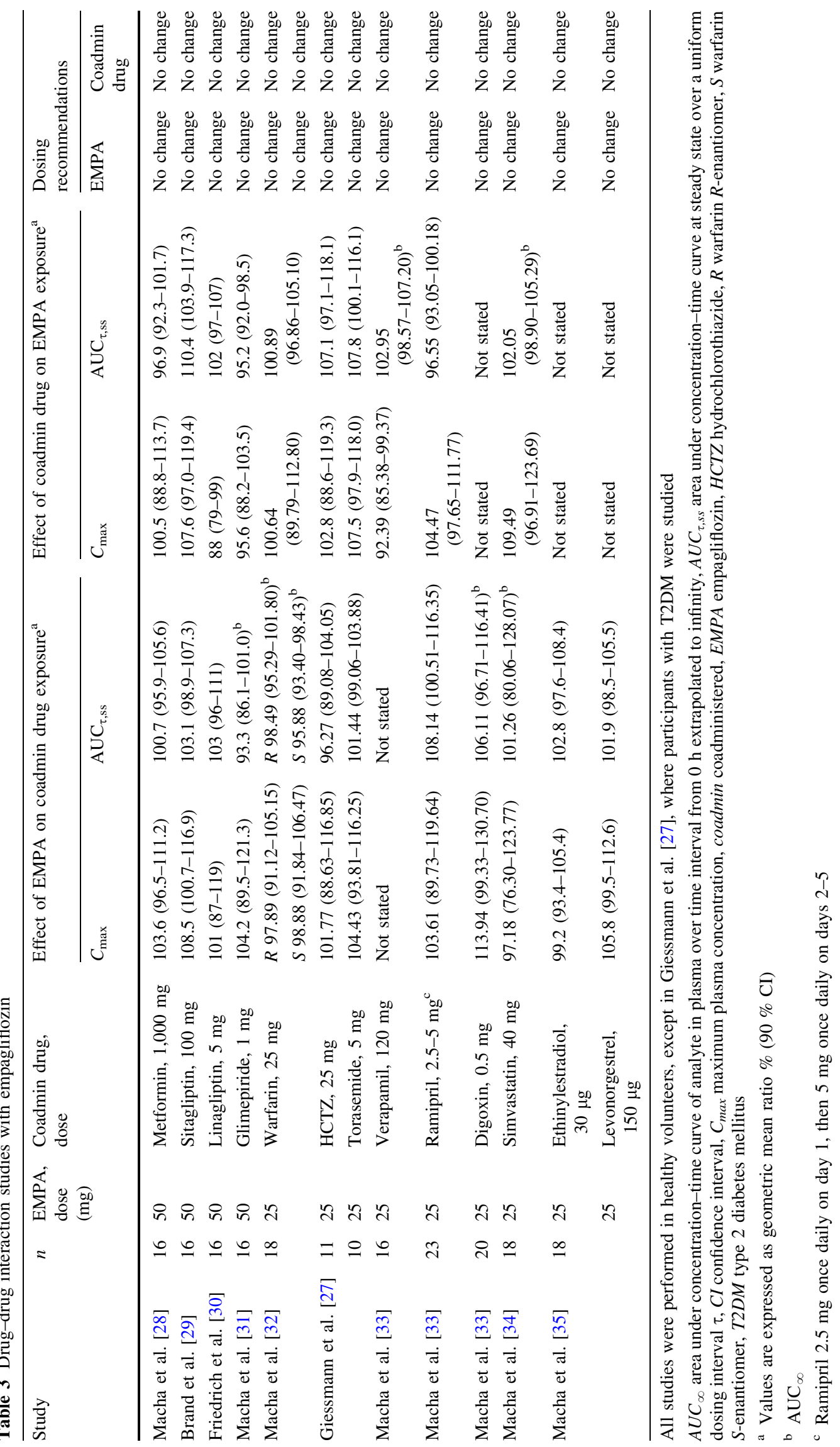


had no effect on the pharmacokinetics of warfarin ( $S$ - or $R$-enantiomers) [32]. Empagliflozin also had no clinically relevant effects on the anticoagulant activity of warfarin [international normalised ratio (INR) GMR $(95 \% \mathrm{CI})$ for peak INR: $0.87(0.73,1.04)$; AUC from 0 to $168 \mathrm{~h}: 0.88$ $(0.79,0.98)][32]$. In addition, the peak anticoagulant effect at $36 \mathrm{~h}$ was similar when warfarin was administered alone and with empagliflozin, and had returned to baseline levels at $96 \mathrm{~h}$ in both groups [32]. As no drugdrug interactions were observed between empagliflozin and warfarin, it was concluded that empagliflozin and warfarin could be coadministered without dosage adjustments of either drug.

\subsection{Coadministration with Cardiovascular/ Antihypertensive Compounds}

\subsubsection{Diuretics (Hydrochlorothiazide or Torasemide)}

A randomised, open-label, crossover study was conducted in patients with T2DM (treated with metformin monotherapy for at least 12 weeks) to investigate potential drugdrug interactions between empagliflozin and hydrochlorothiazide or torasemide [27]. Pharmacokinetic parameters for empagliflozin were similar when empagliflozin was administered alone and coadministered with hydrochlorothiazide or torasemide [27]. Coadministration with hydrochlorothiazide or torasemide had no effect on steady-state exposure of empagliflozin, based on the bioequivalence criteria of $0.80-1.25$ [27]. Equally, pharmacokinetic parameters for hydrochlorothiazide and for torasemide were similar when either drug was administered alone and coadministered with empagliflozin [27]. The study concluded that empagliflozin and hydrochlorothiazide or torasemide could be coadministered without dose adjustments. Interestingly, no adverse events were reported when combining empagliflozin with a strong loop diuretic in this population with T2DM.

\subsubsection{Verapamil}

Verapamil is an inhibitor of P-glycoprotein (P-gp) and empagliflozin is a P-gp substrate; therefore, a randomised, open-label, crossover study was conducted in healthy volunteers (male and female) to investigate potential drugdrug interactions between empagliflozin and verapamil [33]. Subjects were randomised to receive single-dose empagliflozin $25 \mathrm{mg}$ alone or coadministered with singledose verapamil $120 \mathrm{mg}$. Exposure of empagliflozin was not affected by coadministration with verapamil [33]. The lack of drug-drug interaction between empagliflozin and verapamil indicates there is no relevant effect of P-gp inhibition on the pharmacokinetics of empagliflozin, and no dose adjustment of empagliflozin is required when coadministered with verapamil.

\subsubsection{Ramipril}

Exposure of empagliflozin was not affected by coadministration with ramipril [33], and empagliflozin $25 \mathrm{mg}$ once daily had no clinically relevant effect on exposure of ramipril or its active metabolite ramiprilat [33]. Thus, no dose adjustment of ramipril is required when coadministered with empagliflozin, and no dose adjustment of empagliflozin is required when coadministered with ramipril.

\subsubsection{Digoxin}

Digoxin is a P-gp substrate used to examine the effects of P-gp inhibition. A randomised, open-label, crossover study was conducted in healthy volunteers to investigate potential drug-drug interactions between empagliflozin and digoxin [33]. Subjects were randomised to receive singledose digoxin $0.5 \mathrm{mg}$ alone, or empagliflozin $25 \mathrm{mg}$ once daily for 8 days with single-dose digoxin $0.5 \mathrm{mg}$ on day 5 . Coadministration of empagliflozin had no clinically meaningful effect on digoxin exposure (Table 3) [33]. Although a slight increase in digoxin $C_{\max }$ was observed, the authors reported that it was not considered clinically relevant (GMR 113.94; 90 \% CI 99.33-130.70) [33]. The lack of meaningful drug-drug interaction between empagliflozin and digoxin indicates that empagliflozin has no relevant effect on the pharmacokinetics of drugs that are P-gp substrates, and no dose adjustment of digoxin is required when coadministered with empagliflozin.

\subsection{Coadministration with Cholesterol-Lowering Agent (Simvastatin)}

Simvastatin $40 \mathrm{mg}$ had no clinically relevant effect on the pharmacokinetic parameters of empagliflozin [34]. Empagliflozin $25 \mathrm{mg}$ had no clinically relevant effect on the pharmacokinetics of simvastatin or its active metabolite [34]. No dosage adjustments are required for either drug when empagliflozin and simvastatin are coadministered.

\subsection{Coadministration with Oral Contraceptive (Ethinylestradiol/Levonorgestrel)}

The pharmacokinetics of ethinylestradiol and levonorgestrel were unaffected by coadministration with empagliflozin [35]. The $90 \%$ CIs were within the standard bioequivalence boundaries of 80-125\% [35]. Therefore, the study conclusion was that empagliflozin and ethinylestradiol $(30 \mu \mathrm{g}) /$ levonorgestrel $(150 \mu \mathrm{g})$ could be coadministered without the need for dose adjustment. 


\section{Overall Evaluation}

Like the two SGLT2 inhibitors already available (dapagliflozin and canagliflozin) [36], empagliflozin has favourable pharmacokinetic properties. Empagliflozin has an excellent oral bioavailability, a rather long $t_{1 / 2}$ allowing once-daily administration, no active metabolites and a very limited (if any) propensity to drug-drug interactions. Furthermore, long-term administration showed a rather limited accumulation index and $\mathrm{CL} / F$ at steady state was similar to corresponding single-dose values, suggesting linear pharmacokinetics with respect to time. The reported $C_{R}$ of empagliflozin was higher than that reported in other studies with dapagliflozin and canagliflozin and the percentage of empagliflozin excreted as unchanged parent compound in urine (approximately $18 \%$ ) was also higher than the corresponding percentages reported with the two other SGLT2 inhibitors $(<3 \%)$ [36]. However, the clinical significance of this finding remains unclear. No clinically relevant changes in pharmacokinetic parameters were observed in patients with T2DM [22, 23], compared with healthy volunteers [19], or in patients with mild/moderate renal impairment [24], or hepatic impairment [26]. UGE increased linearly with exposure to the drug up to a daily dose of $100 \mathrm{mg}$ in healthy volunteers [19]. However, in patients with T2DM, the UGE rate was not enhanced further when the dosage was increased from 25 to $100 \mathrm{mg}$ [23], which suggests a maximal dose of $25 \mathrm{mg}$ is recommended for phase III clinical trials and for future use in clinical practice. As with other SGLT2 inhibitors, and because of the specific mechanism of action, the pharmacodynamic effect of empagliflozin in promoting UGE, and thus in reducing hyperglycaemia in patients with T2DM, was diminished with the reduction in glomerular filtration rate [24]. This would imply that renal function should be carefully assessed before considering the use of empagliflozin in patients with T2DM, and that renal function should be monitored with long-term use of this SGLT2 inhibitor, as is also recommended for dapagliflozin and canagliflozin. To date, there are no head-to-head clinical trials comparing the pharmacokinetic/pharmacodynamic properties of these three SGLT2 inhibitors. Several phase III clinical trials with empagliflozin as monotherapy or in combination with other glucose-lowering agents for the management of T2DM are already available [13-17], with apparently similar efficacy/safety data to dapagliflozin and canagliflozin [37]. However, direct comparative clinical studies would be of interest in the future. Finally, considering the vast panel of glucoselowering drugs already available for the management of T2DM, the best positioning of SGLT2 inhibitors in general, and of empagliflozin in particular, remains to be more precisely defined. Large ongoing trials, including two focusing on empagliflozin [38,39], will help to better evaluate the benefit/risk balance of this new pharmacological class [36].

\section{Summary and Conclusions}

This review summarises the pharmacokinetic and pharmacodynamic studies performed in humans using the SGLT2 inhibitor empagliflozin. SGLT2 inhibitors, including empagliflozin, are the first pharmacological class of antidiabetes agents to target the kidney. By stimulating UGE, empagliflozin and the other SGLT2 inhibitors are able to improve glucose control together with promoting weight loss and reducing systolic blood pressure. Unlike other oral antidiabetes agents currently available, SGLT2 inhibitors, including empagliflozin, do not stimulate insulin secretion and, thus, are not associated with the risk of hypoglycaemia. Furthermore, the selectivity of these agents for SGLT2 over SGLT1 reduces the incidence of gastrointestinal side effects. The mechanism of action of SGLT2 inhibitors permits their effective use in all stages of T2DM, as monotherapy, as add-on to other glucose-lowering agents, and as add-on to insulin.

The pharmacologically noteworthy difference between individual SGLT2 inhibitors may be in the selectivity of SGLT2 versus SGLT1; however, other potential differences remain unknown due to the absence of head-to-head trials. Nevertheless, current safety data imply that SGLT2 inhibitors have an acceptable safety profile and are welltolerated, despite a small increase in genital infections and urinary tract infections.

In conclusion, based on the available data, empagliflozin appears to be a promising option for patients with T2DM, and offers the possibility of administration with other oral antidiabetes drugs.

Acknowledgments The author was fully responsible for all content and editorial decisions, was involved at all stages of manuscript development, and has approved the final version of the manuscript that reflects the author's interpretation and conclusions. Medical writing assistance, supported financially by Boehringer Ingelheim, was provided by Debra Brocksmith, $\mathrm{MB} \mathrm{ChB}, \mathrm{PhD}$, of Envision Scientific Solutions, during the preparation of this manuscript. Boehringer Ingelheim was given the opportunity to check the data used in the manuscript for factual accuracy only.

André J. Scheen has received grants from Novo Nordisk. He has also received consulting fees from Novo Nordisk, Eli Lilly, SanofiAventis, Servier, Boehringer Ingelheim, MSD, AstraZeneca, BMS, Novartis and Takeda.

Open Access This article is distributed under the terms of the Creative Commons Attribution Noncommercial License which permits any noncommercial use, distribution, and reproduction in any medium, provided the original author(s) and the source are credited. 


\section{References}

1. Wright EM, Loo DD, Hirayama BA. Biology of human sodium glucose transporters. Physiol Rev. 2011;91:733-94.

2. Hediger MA, Rhoads DB. Molecular physiology of sodiumglucose cotransporters. Physiol Rev. 1994;74:993-1026.

3. Abdul-Ghani MA, Norton L, Defronzo RA. Role of sodiumglucose cotransporter 2 (SGLT 2) inhibitors in the treatment of type 2 diabetes. Endocr Rev. 2011;32:515-31.

4. Bolinder J, Ljunggren $\mathrm{O}$, Kullberg $\mathrm{J}$, et al. Effects of dapagliflozin on body weight, total fat mass, and regional adipose tissue distribution in patients with type 2 diabetes mellitus with inadequate glycemic control on metformin. J Clin Endocrinol Metab. 2012;97:1020-31.

5. Toubro S, Cefalu WT, Xie J, et al. Canagliflozin, a sodium glucose co-transporter 2 inhibitor, reduces body weight mainly through loss of fat mass in subjects with type 2 diabetes [abstract no. 762]. Diabetologia. 2012;55:S313.

6. Bristol Myers Squibb-AstraZeneca EEIG. Summary of product characteristics: Forxiga $5 \mathrm{mg} \& 10 \mathrm{mg}$ film coated tablets. Electronic Medicines Compendium. http://www.medicines.org. uk/emc/medicine/27188/SPC/Forxiga +5\&thinsp;mg++\%26+ 10+mg+film+coated+tablets/. Accessed 17 Jul 2013.

7. Janssen Pharmaceuticals Inc. INVOKANA ${ }^{\mathrm{TM}}$ (canagliflozin) tablets, for oral use [prescribing information] (issued March 2013). http://www.invokanahcp.com/prescribing-information. pdf. Accessed 17 Jul 2013.

8. Grempler R, Thomas L, Eckhardt M, et al. Empagliflozin, a novel selective sodium glucose cotransporter-2 (SGLT-2) inhibitor: characterisation and comparison with other SGLT-2 inhibitors. Diabetes Obes Metab. 2012;14:83-90.

9. Polidori D, Sha S, Mudaliar S, et al. Canagliflozin lowers postprandial glucose and insulin by delaying intestinal glucose absorption in addition to increasing urinary glucose excretion: results of a randomized, placebo-controlled study. Diabetes Care. 2013;36:2154-61.

10. Zambrowicz B, Ding ZM, Ogbaa I, et al. Effects of LX4211, a dual SGLT1/SGLT2 inhibitor, plus sitagliptin on postprandial active GLP-1 and glycemic control in type 2 diabetes. Clin Ther. 2013;35:273-85.

11. Powell DR, Smith M, Greer J, et al. LX4211 increases serum glucagon-like peptide 1 and peptide YY levels by reducing sodium/glucose cotransporter 1 (SGLT1)-mediated absorption of intestinal glucose. J Pharmacol Exp Ther. 2013;345:250-9.

12. Thomas L, Grempler R, Eckhardt M, et al. Long-term treatment with empagliflozin, a novel, potent and selective SGLT-2 inhibitor, improves glycaemic control and features of metabolic syndrome in diabetic rats. Diabetes Obes Metab. 2012;14:94-6.

13. Häring HU, Merker L, Seewaldt-Becker E, et al. Empagliflozin as add-on to metformin for 24 weeks improves glycemic control in patients with type 2 diabetes (T2DM) [abstract no. 1092-P]. Diabetes. 2013;62 Suppl 1:A282.

14. Häring HU, Merker L, Seewaldt-Becker E, et al. Empagliflozin as add-on to metformin plus sulfonylurea in patients with type 2 diabetes: a 24-week, randomized, double-blind, placebo-controlled trial. Diabetes Care. 2013;36:3396-404. doi:10.2337/ dc12-2673.

15. Kovacs CS, Seshiah V, Swallow R, et al. Empagliflozin improves glycaemic and weight control as add-on therapy to pioglitazone or pioglitazone plus metformin in patients with type 2 diabetes: a 24-week, randomized, placebo-controlled trial. Diabetes Obes Metab. Epub 2013 Aug 1. doi:10.1111/dom.12188.

16. Roden M, Weng J, Eilbracht J, et al. Empagliflozin monotherapy with sitagliptin as an active comparator in patients with type 2 diabetes: a randomised, double-blind, placebo-controlled, phase 3 trial. Lancet Diabetes Endocrinol. 2013;1:208-19. doi:10.1016/ S2213-8587(13)70084-6.

17. Rosenstock J, Jelaska A, Kim G, et al. Empagliflozin as add-on to basal insulin for 78 weeks improves glycemic control with weight loss in insulin-treated (T2DM) [abstract no. 1102-P]. Diabetes. 2013;62 Suppl 1:A285.

18. Kim G, Gerich JE, Salsali A, et al. Empagliflozin (EMPA) increases genital infections but not urinary tract infections (UTIs) in pooled data from four pivotal phase III trials [abstract no. 74-LB]. Diabetes. 2013;62 Suppl 1:LB21.

19. Seman L, Macha S, Nehmiz G, et al. Empagliflozin (BI 10773), a potent and selective SGLT2 inhibitor, induces dose-dependent glucosuria in healthy subjects. Clin Pharmacol Drug Dev. 2013;2:152-61.

20. Sarashina A, Koiwai K, Seman LJ, et al. Safety, tolerability, pharmacokinetics and pharmacodynamics of single doses of empagliflozin, a sodium glucose cotransporter 2 (SGLT2) inhibitor, in healthy Japanese subjects. Drug Metab Pharmacokinet. 2013;28:213-9.

21. Macha S, Jungnik A, Hohl K, et al. Effect of food on the pharmacokinetics of empagliflozin, a sodium glucose cotransporter 2 (SGLT2) inhibitor, and assessment of dose proportionality in healthy volunteers. Int J Clin Pharmacol Ther. 2013;51:873-9.

22. Heise T, Seman L, Macha S, et al. Safety, tolerability, pharmacokinetics, and pharmacodynamics of multiple rising doses of empagliflozin in patients with type 2 diabetes mellitus. Diabetes Ther. Epub 2013 Jul 10. doi:10.1007/s13300-013-0030-2.

23. Heise T, Seewaldt-Becker E, Macha S, et al. Safety, tolerability, pharmacokinetics and pharmacodynamics following 4 weeks' treatment with empagliflozin once daily in patients with type 2 diabetes. Diabetes Obes Metab. 2013;15:613-21.

24. Macha S, Mattheus M, Halabi A, et al. Pharmacokinetics, pharmacodynamics and safety of empagliflozin, a sodium glucose cotransporter 2 (SGLT2) inhibitor, in subjects with renal impairment. Diabetes Obes Metab. Epub 2013 Jul 16. doi:10. 1111/dom.12182.

25. Scheen AJ. Pharmacokinetic considerations for the treatment of diabetes in patients with chronic kidney disease. Expert Opin Drug Metab Toxicol. 2013;9:529-50. doi:10.1517/17425255. 2013.777428.

26. Macha S, Rose P, Mattheus M, et al. Pharmacokinetics, safety and tolerability of empagliflozin, a sodium glucose cotransporter 2 inhibitor, in patients with hepatic impairment. Diabetes Obes Metab. Epub 2013 Jul 16. doi:10.1111/dom.12183.

27. Giessmann T, Heise $T$, Macha S, et al. Lack of interaction between the sodium glucose cotransporter-2 inhibitor empagliflozin and hydrochlorothiazide or torasemide in patients with T2DM [abstract no. 2440-PO]. Diabetes. 2012;61:A614.

28. Macha S, Dieterich S, Mattheus M, et al. Pharmacokinetics of empagliflozin, a sodium glucose cotransporter-2 (SGLT2) inhibitor, and metformin following co-administration in healthy volunteers. Int J Clin Pharmacol Ther. 2013;51:132-40.

29. Brand T, Macha S, Mattheus M, et al. Pharmacokinetics of empagliflozin, a sodium glucose cotransporter-2 (SGLT-2) inhibitor, coadministered with sitagliptin in healthy volunteers. Adv Ther. 2012;29:889-99.

30. Friedrich C, Metzmann K, Rose P, et al. A randomized, openlabel, crossover study to evaluate the pharmacokinetics of empagliflozin and linagliptin after coadministration in healthy male volunteers. Clin Ther. 2013;35:A33-42.

31. Macha S, Mattheus M, Pinnetti S, et al. Pharmacokinetics of empagliflozin, a sodium glucose cotransporter 2 inhibitor, and glimepiride following co-administration in healthy volunteers: a randomised, open-label, crossover study. Diabetes Res Clin Metab. 2012;1:1-7. 
32. Macha S, Rose P, Mattheus M, et al. Lack of drug-drug interaction between empagliflozin, a sodium glucose cotransporter 2 inhibitor, and warfarin in healthy volunteers. Diabetes Obes Metab. 2013;15:316-23.

33. Macha S, Sennewald R, Rose P, et al. Lack of clinically relevant drug-drug interaction between empagliflozin, a sodium glucose cotransporter 2 inhibitor, and verapamil, ramipril, or digoxin in healthy volunteers. Clin Ther. 2013;35:226-35.

34. Macha S, Lang B, Pinnetti S, et al. Lack of pharmacokinetic interaction between the sodium glucose cotransporter-2 (SGLT2) inhibitor empagliflozin and simvastatin in healthy volunteers [abstract no. PCS-33-7]. J Diabetes Investig. 2012;3:228.

35. Macha S, Mattheus M, Pinnetti S, et al. Effect of empagliflozin on the steady-state pharmacokinetics of ethinylestradiol and levonorgestrel in healthy female volunteers. Clin Drug Investig. 2013;33:351-7.
36. Scheen AJ. Evaluating SGLT-2 inhibitors for type 2 diabetes: pharmacokinetic and toxicological considerations. Expert Opin Drug Metab Toxicol. 2013 (in press).

37. Vasilakou D, Karagiannis T, Athanasiadou E, et al. Sodium-glucose cotransporter 2 inhibitors for type 2 diabetes: a systematic review and meta-analysis. Ann Intern Med. 2013;159:262-74. doi:10.7326/ 0003-4819-159-4-201308200-00007.

38. Inzucchi SE, Zinman B, Lachin JM, et al. Design of the empagliflozin cardiovascular outcome event trial in type 2 diabetes mellitus [abstract no. 944]. Diabetologia. 2013;56:S378.

39. Ridderstråle M, Svaerd R, Zeller C, et al. Rationale, design and baseline characteristics of a 4-year (208-week) phase III trial of empagliflozin, an SGLT2 inhibitor, versus glimepiride as add-on to metformin in patients with type 2 diabetes mellitus with insufficient glycemic control. Cardiovasc Diabetol. 2013;12:129. doi:10.1186/1475-2840-12-129. 